\title{
A Comprehensive Investigation of Chitosan/tripolyphosphate Nanoparticles Using Artificial Neural Networks
}

mona akbari

Hormozgan University https://orcid.org/0000-0002-1553-1424

Maryam Akbari

Iran University of Science and Technology

Zohreh Rahimi ( $\nabla$ rahimizus@yahoo.com)

Kermanshah University of Medical Sciences

\section{Research Article}

Keywords: Chitosan, Tripolyphosphate, Nanoparticles, Artificial neural networks model, Estimation, Drug Delivery

Posted Date: February 18th, 2021

DOI: https://doi.org/10.21203/rs.3.rs-197903/v1

License: () (i) This work is licensed under a Creative Commons Attribution 4.0 International License. Read Full License 


\section{Abstract}

Chitosan/tripolyphosphate (CS/TPP) nanoparticles have been widely investigated in many applications. Many experimental studies evaluated effective parameters on CS/TPP nanoparticles without a comprehensive study to explain the influence of parameters on nanoparticles. The purpose of present work was to build a mathematical model capable of predicting the particle size and zeta potential of CS/TPP nanoparticles using seven significant factors, including CS and TPP concentrations, deacetylation degree of CS (DD), the molecular weight of CS, pH of CS solution and temperature, and their interactions on the size and zeta potential of CS/TPP nanoparticles. A model was built using artificial neural network including properties of nanoparticles as input with particle size and zeta potential as output. Artificial neural networks (ANN) models were used based on 8 experimental works consisting 160 data to estimate the variation tendency of size and zeta potential. The established model successfully predicted particle size and zeta potential of nanoparticles covering a range of 50-1000 $\mathrm{nm}$. All parameters had significant effects on the size, the interaction between parameters changed the relationship pattern between them. In addition, results indicated that the main reason for the unexplained difference in previous works is the interactions between parameters. In addition, there is a relationship between size and zeta potential, which is due to the attractive and repulsive electrostatic charges, ionic interactions, CS chain length and viscosity. The ANN models in this work were valid for other papers.

\section{Full Text}

This preprint is available for download as a PDF.

\section{Tables}

Table 1 Employed references for this work

\section{Name of reference}

Authors

1 Modulation of surface charge, particle size and morphological properties of chitosan-TPP nanoparticles intended for gene delivery

Gan et al. [24]

2 Formation mechanism of monodisperse, low molecular weight chitosan nanoparticles by ionic gelation technique Fan et al. ${ }^{[31]}$

3 Depolymerized Chitosan Nanoparticles for Protein Delivery: Preparation and Characterization Janes et al. [44]

4 Chitosan-tripolyphosphate nanoparticles: Optimization of formulation parameters for improving process yield at a novel $\mathrm{pH}$ using artificial neural networks

Hashad et al. [25]

5 Preparation, characterization and evaluation of selenite-loaded chitosan/TPP nanoparticles with or without zein coating Luo et al. ${ }^{[29]}$

6 Preparation and properties of ionically cross-linked chitosan nanoparticles Liu \& Gao ${ }^{[27]}$

7 Antifungal Activity of Chitosan Nanoparticles and Correlation with Their Physical Properties Ing et al. [28]

8 Chitosan nanoparticles as a novel delivery system for ammonium glycyrrhizinate

Wu et al. ${ }^{[36]}$

Table 2 Range of employed data.

\begin{tabular}{|lll|}
\hline Parameters & Range & Set point \\
\hline CS concentration $(\mathrm{mg} / \mathrm{ml})$ & $0.5-9$ & 1 \\
\hline TPP concentration $(\mathrm{mg} / \mathrm{ml})$ & $0.1-2.91$ & 0.5 \\
\hline CS molecular weight $(\mathrm{kDa})$ & $6.632-620$ & 50 \\
\hline Degree of deacetylation $(\%)$ & $80-95$ & 87 \\
\hline CS/TPP mass ratio $($ dimensionless $)$ & $2.5-9$ & 5 \\
\hline pH of CS solution & $1-6.2$ & 4.7 \\
\hline CS temperature $\left({ }^{\circ} \mathrm{C}\right)$ & $20-60$ & 25 \\
\hline
\end{tabular}

Table 3 Experimental and predicted NPs' size and zeta potential for "training", "testing" and "validation" data. 


\begin{tabular}{|c|c|c|c|c|c|c|c|}
\hline \multirow[t]{2}{*}{ Sample number } & \multicolumn{3}{|c|}{ NP size $(\mathrm{nm})$} & \multicolumn{2}{|c|}{ Sample number } & \multicolumn{2}{|c|}{ Zeta potential $(\mathrm{mV})$} \\
\hline & Experimental & Predicted & Error & & Experimental & Predicted & Error \\
\hline 1 & 196 & 191.392 & 4.608 & 1 & 25 & 24.915 & 0.085 \\
\hline 2 & 255 & 194.9054 & 60.0946 & 2 & 26.1 & 26.1931 & -0.0931 \\
\hline 3 & 259 & 200.0812 & 58.9188 & 3 & 23.9 & 23.0341 & 0.8659 \\
\hline 4 & 220 & 233.6774 & -13.6774 & 4 & 23.3 & 24.6697 & -1.3697 \\
\hline 5 & 253 & 238.9213 & 14.0787 & 5 & 26 & 25.8526 & 0.1474 \\
\hline 6 & 245 & 244.4032 & 0.5968 & 6 & 43.3 & 43.693 & -0.393 \\
\hline 7 & 276 & 322.0349 & -46.0349 & 7 & 45.5 & 45.5591 & -0.0591 \\
\hline 8 & 337 & 322.2837 & 14.7163 & 8 & 47.6 & 46.5735 & 1.0265 \\
\hline 9 & 335 & 322.1547 & 12.8453 & 9 & 45.9 & 46.3906 & -0.4906 \\
\hline 10 & 358 & 320.4057 & 37.5943 & 10 & 24 & 23.0341 & 0.9659 \\
\hline 11 & 219 & 233.6774 & -14.6774 & 11 & 23.5 & 24.6697 & -1.1697 \\
\hline 12 & 254 & 238.9213 & 15.0787 & 12 & 26 & 25.8526 & 0.1474 \\
\hline 13 & 247 & 244.4032 & 2.5968 & 13 & 27 & 26.7377 & 0.2623 \\
\hline 14 & 253 & 258.9107 & -5.9107 & 14 & 39.6 & 36.9604 & 2.6396 \\
\hline 15 & 172.6341 & 170.2033 & 2.4308 & 15 & 38.6 & 37.6109 & 0.9891 \\
\hline 16 & 165.0244 & 162.6582 & 2.3662 & 16 & 38 & 37.6501 & 0.3499 \\
\hline 17 & 160.5854 & 156.7379 & 3.8475 & 17 & 36.7 & 37.2112 & -0.5112 \\
\hline 18 & 149.1707 & 152.1048 & -2.9341 & 18 & 36.4 & 36.4185 & -0.0185 \\
\hline 19 & 144.7317 & 148.4936 & -3.7619 & 19 & 35 & 35.3787 & -0.3787 \\
\hline 20 & 137.7561 & 145.6965 & -7.9404 & 20 & 34.05 & 34.1809 & -0.1309 \\
\hline 21 & 135.2195 & 143.5508 & -8.3313 & 21 & 33.5 & 32.8986 & 0.6014 \\
\hline 22 & 135.8537 & 141.9284 & -6.0747 & 22 & 32.4 & 31.59 & 0.81 \\
\hline 23 & 133.3171 & 140.7284 & -7.4113 & 23 & 30 & 30.2991 & -0.2991 \\
\hline 24 & 148.5366 & 139.8704 & 8.6662 & 24 & 26 & 29.0576 & -3.0576 \\
\hline 25 & 237.3171 & 139.2902 & 98.0269 & 25 & 50.13889 & 49.826 & 0.312889 \\
\hline 26 & 150.3371 & 158.8149 & -8.4778 & 26 & 47.91667 & 48.5997 & -0.68303 \\
\hline 27 & 167.5281 & 163.4473 & 4.0808 & 27 & 46.94444 & 47.1095 & -0.16506 \\
\hline 28 & 168.5393 & 170.092 & -1.5527 & 28 & 46.66667 & 45.351 & 1.315667 \\
\hline 29 & 176.6292 & 179.948 & -3.3188 & 29 & 43.75 & 43.3394 & 0.4106 \\
\hline 30 & 184.7191 & 195.4498 & -10.7307 & 30 & 42.77778 & 41.1081 & 1.669678 \\
\hline 31 & 193.8202 & 220.901 & -27.0808 & 31 & 48.75 & 48.0715 & 0.6785 \\
\hline 32 & 159.4382 & 172.4194 & -12.9812 & 32 & 46.25 & 45.7064 & 0.5436 \\
\hline 33 & 173.5955 & 172.1487 & 1.4468 & 33 & 42.5 & 42.9819 & -0.4819 \\
\hline 34 & 178.6517 & 174.2484 & 4.4033 & 34 & 41.52778 & 39.9952 & 1.532578 \\
\hline 35 & 188.764 & 179.867 & 8.897 & 35 & 39.58333 & 36.8655 & 2.717833 \\
\hline 36 & 202.9213 & 191.3284 & 11.5929 & 36 & 44.30556 & 44.3411 & -0.03554 \\
\hline 37 & 178.6517 & 178.3189 & 0.3328 & 37 & 35 & 34.9762 & 0.0238 \\
\hline 38 & 250.4494 & 211.8399 & 38.6095 & 38 & 48.3 & 45.6423 & 2.6577 \\
\hline 39 & 136.2 & 144.7588 & -8.5588 & 39 & 44.2 & 43.5762 & 0.6238 \\
\hline 40 & 142.3 & 140.2824 & 2.0176 & 40 & 41 & 41.4341 & -0.4341 \\
\hline 41 & 152.9 & 142.9023 & 9.9977 & 41 & 39.7 & 39.2897 & 0.4103 \\
\hline 42 & 171.2 & 154.8362 & 16.3638 & 42 & 37.3 & 37.2066 & 0.0934 \\
\hline
\end{tabular}




\begin{tabular}{|c|c|c|c|c|c|c|c|}
\hline 43 & 190.3 & 180.5335 & 9.7665 & 43 & 33.3 & 33.3747 & -0.0747 \\
\hline 44 & 312.7 & 309.0546 & 3.6454 & 44 & 31.6 & 31.639 & -0.039 \\
\hline 45 & 429.6 & 431.6663 & -2.0663 & 45 & 28.2 & 28.4072 & -0.2072 \\
\hline 46 & 712.4 & 723.6779 & -11.2779 & 46 & 26.9 & 26.8353 & 0.0647 \\
\hline 47 & 800 & 825.2271 & -25.2271 & 47 & 25.2 & 25.2536 & -0.0536 \\
\hline 48 & 900 & 887.45 & 12.55 & 48 & 43.9 & 43.3508 & 0.5492 \\
\hline 49 & 145.3 & 142.1561 & 3.1439 & 49 & 40.3 & 40.7971 & -0.4971 \\
\hline 50 & 150.5 & 149.6152 & 0.8848 & 50 & 39.1 & 38.1255 & 0.9745 \\
\hline 51 & 165.2 & 166.7953 & -1.5953 & 51 & 37.2 & 35.4157 & 1.7843 \\
\hline 52 & 182.3 & 197.1717 & -14.8717 & 52 & 37.7 & 37.9471 & -0.2471 \\
\hline 53 & 155 & 149.7363 & 5.2637 & 53 & 34.8 & 34.5576 & 0.2424 \\
\hline 54 & 188.9 & 171.5868 & 17.3132 & 54 & 49.2 & 48.602 & 0.598 \\
\hline 55 & 143.2 & 152.58 & -9.38 & 55 & 46.8 & 47.3145 & -0.5145 \\
\hline 56 & 152.1 & 152.3497 & -0.2497 & 56 & 45.6 & 45.84 & -0.24 \\
\hline 57 & 159.2 & 156.8669 & 2.3331 & 57 & 44.3 & 44.2108 & 0.0892 \\
\hline 58 & 172.8 & 168.6003 & 4.1997 & 58 & 42.7 & 42.4832 & 0.2168 \\
\hline 59 & 181.9 & 191.366 & -9.466 & 59 & 39.4 & 39.0259 & 0.3741 \\
\hline 60 & 273.2 & 293.5664 & -20.3664 & 60 & 37.1 & 37.4327 & -0.3327 \\
\hline 61 & 387.2 & 381.6207 & 5.5793 & 61 & 36.8 & 35.9872 & 0.8128 \\
\hline 62 & 519.6 & 485.676 & 33.924 & 62 & 34.2 & 34.6968 & -0.4968 \\
\hline 63 & 604 & 587.5001 & 16.4999 & 63 & 33.3 & 33.5423 & -0.2423 \\
\hline 64 & 692.1 & 673.518 & 18.582 & 64 & 32.7 & 32.4857 & 0.2143 \\
\hline 65 & 726.6 & 741.3184 & -14.7184 & 65 & 30.8 & 30.48 & 0.32 \\
\hline 66 & 846.7 & 838.0157 & 8.6843 & 66 & 30 & 29.4489 & 0.5511 \\
\hline 67 & 858.9 & 873.6001 & -14.7001 & 67 & 28.5 & 28.3657 & 0.1343 \\
\hline 68 & 893.6 & 902.4185 & -8.8185 & 68 & 26.7 & 27.2266 & -0.5266 \\
\hline 69 & 908.7 & 924.8605 & -16.1605 & 69 & 26.2 & 26.0438 & 0.1562 \\
\hline 70 & 938.7 & 941.5162 & -2.8162 & 70 & 46.8 & 46.971 & -0.171 \\
\hline 71 & 156.1 & 155.1256 & 0.9744 & 71 & 44.4 & 44.6003 & -0.2003 \\
\hline 72 & 163.7 & 155.9636 & 7.7364 & 72 & 40.3 & 42.1262 & -1.8262 \\
\hline 73 & 170.7 & 163.4231 & 7.2769 & 73 & 39.8 & 39.7173 & 0.0827 \\
\hline 74 & 181.5 & 179.1901 & 2.3099 & 74 & 37.8 & 37.5069 & 0.2931 \\
\hline 75 & 192.2 & 206.4231 & -14.2231 & 75 & 33.8 & 33.91 & -0.11 \\
\hline 76 & 310.2 & 318.1746 & -7.9746 & 76 & 32.7 & 32.5017 & 0.1983 \\
\hline 77 & 427.5 & 413.6756 & 13.8244 & 77 & 32.1 & 31.2852 & 0.8148 \\
\hline 78 & 546.3 & 529.2565 & 17.0435 & 78 & 30.4 & 30.1968 & 0.2032 \\
\hline 79 & 654.3 & 644.1477 & 10.1523 & 79 & 29 & 29.179 & -0.179 \\
\hline 80 & 721.5 & 739.4754 & -17.9754 & 80 & 28.6 & 28.1878 & 0.4122 \\
\hline 81 & 783.2 & 810.1916 & -26.9916 & 81 & 27.5 & 27.196 & 0.304 \\
\hline 82 & 848.3 & 860.7398 & -12.4398 & 82 & 25.3 & 26.1929 & -0.8929 \\
\hline 83 & 881 & 897.0587 & -16.0587 & 83 & 25.1 & 25.1817 & -0.0817 \\
\hline 84 & 936.8 & 923.3845 & 13.4155 & 84 & 24.8 & 24.1746 & 0.6254 \\
\hline 85 & 988.6 & 942.3268 & 46.2732 & 85 & 41.3 & 38.4777 & 2.8223 \\
\hline 86 & 162.7 & 158.6043 & 4.0957 & 86 & 37.4 & 37.4541 & -0.0541 \\
\hline
\end{tabular}




\begin{tabular}{|c|c|c|c|c|c|c|c|}
\hline 87 & 176.1 & 167.7879 & 8.3121 & 87 & 33.2 & 33.6508 & -0.4508 \\
\hline 88 & 257 & 294.1863 & -37.1863 & 88 & 32.6 & 32.6465 & -0.0465 \\
\hline 89 & 362.3 & 368.0349 & -5.7349 & 89 & 31.8 & 31.5815 & 0.2185 \\
\hline 90 & 478 & 468.344 & 9.656 & 90 & 29.5 & 29.2775 & 0.2225 \\
\hline 91 & 697.3 & 702.8856 & -5.5856 & 91 & 26.3 & 26.8587 & -0.5587 \\
\hline 92 & 828 & 860.1881 & -32.1881 & 92 & 25.9 & 25.6697 & 0.2303 \\
\hline 93 & 898.5 & 902.948 & -4.448 & 93 & 23.2 & 24.5275 & -1.3275 \\
\hline 94 & 976.8 & 931.0267 & 45.7733 & 94 & 14.43 & 15.9199 & -1.4899 \\
\hline 95 & 166.48 & 256.0483 & -89.5683 & 95 & 15.89 & 16.109 & -0.219 \\
\hline 96 & 252 & 268.8835 & -16.8835 & 96 & 16.62 & 16.1109 & 0.5091 \\
\hline 97 & 260.75 & 276.563 & -15.813 & 97 & 16.2 & 15.9545 & 0.2455 \\
\hline 98 & 289.8 & 277.6585 & 12.1415 & 98 & 19.34 & 17.4885 & 1.8515 \\
\hline 99 & 278.35 & 284.1268 & -5.7768 & 99 & 16.71 & 17.7676 & -1.0576 \\
\hline 100 & 317.8 & 316.7133 & 1.0867 & 100 & 17.92 & 17.7017 & 0.2183 \\
\hline 101 & 374.94 & 345.8189 & 29.1211 & 101 & 18.65 & 17.7819 & 0.8681 \\
\hline 102 & 173.13 & 261.7309 & -88.6009 & 102 & 18.7 & 18.8987 & -0.1987 \\
\hline 103 & 386.98 & 314.0635 & 72.9165 & 103 & 20.8 & 19.716 & 1.084 \\
\hline 104 & 420.26 & 371.2113 & 49.0487 & 104 & 19.9 & 20.0569 & -0.1569 \\
\hline 105 & 469.56 & 429.3081 & 40.2519 & 105 & 20.12 & 19.8889 & 0.2311 \\
\hline 106 & 487.56 & 483.7737 & 3.7863 & 106 & 15.8 & 17.1213 & -1.3213 \\
\hline 107 & 162.7 & 165.9455 & -3.2455 & 107 & 18.52 & 17.3425 & 1.1775 \\
\hline 108 & 174.55 & 176.0433 & -1.4933 & 108 & 18.71 & 17.2485 & 1.4615 \\
\hline 109 & 184.03 & 185.2919 & -1.2619 & 109 & 17.54 & 16.9169 & 0.6231 \\
\hline 110 & 200.05 & 193.2886 & 6.7614 & 110 & 18.16 & 18.3356 & -0.1756 \\
\hline 111 & 169.94 & 169.7467 & 0.1933 & 111 & 20.06 & 19.0711 & 0.9889 \\
\hline 112 & 198.57 & 190.205 & 8.365 & 112 & 19.31 & 18.5602 & 0.7498 \\
\hline 113 & 232.7 & 260.3307 & -27.6307 & 113 & 19.82 & 19.2969 & 0.5231 \\
\hline 114 & 184.52 & 192.5311 & -8.0111 & 114 & 20.9 & 21.6878 & -0.7878 \\
\hline 115 & 299.49 & 265.9476 & 33.5424 & 115 & 21.7 & 22.0144 & -0.3144 \\
\hline 116 & 317.39 & 311.2215 & 6.1685 & 116 & 20.4 & 21.6979 & -1.2979 \\
\hline 117 & 331.03 & 361.0367 & -30.0067 & 117 & 17.16 & 18.0042 & -0.8442 \\
\hline 118 & 170.08 & 172.3376 & -2.2576 & 118 & 18.78 & 18.2235 & 0.5565 \\
\hline 119 & 204.1 & 189.0351 & 15.0649 & 119 & 20.01 & 18.0644 & 1.9456 \\
\hline 120 & 217.75 & 206.6799 & 11.0701 & 120 & 17.62 & 17.6373 & -0.0173 \\
\hline 121 & 231.68 & 225.0128 & 6.6672 & 121 & 17.14 & 17.095 & 0.045 \\
\hline 122 & 244.66 & 243.7135 & 0.9465 & 122 & 18.75 & 19.6547 & -0.9047 \\
\hline 123 & 170.58 & 195.2295 & -24.6495 & 123 & 22.3 & 20.5348 & 1.7652 \\
\hline 124 & 207.93 & 218.4398 & -10.5098 & 124 & 19.87 & 20.5911 & -0.7211 \\
\hline 125 & 264.2 & 270.6047 & -6.4047 & 125 & 19.62 & 19.9186 & -0.2986 \\
\hline 126 & 278.28 & 298.7621 & -20.4821 & 126 & 20.21 & 21.1927 & -0.9827 \\
\hline 127 & 205.68 & 210.4188 & -4.7388 & 127 & 24.31 & 23.0349 & 1.2751 \\
\hline 128 & 227.38 & 236.3225 & -8.9425 & 128 & 24.6 & 24.2875 & 0.3125 \\
\hline 129 & 293.25 & 265.3291 & 27.9209 & 129 & 23.4 & 24.7834 & -1.3834 \\
\hline 130 & 300.86 & 297.2615 & 3.5985 & 130 & 25.46 & 24.5356 & 0.9244 \\
\hline
\end{tabular}




\begin{tabular}{|c|c|c|c|c|c|c|c|}
\hline 131 & 312.32 & 331.7607 & -19.4407 & 131 & 45 & 44.903 & 0.097 \\
\hline 132 & 321 & 328.1618 & -7.1618 & 132 & 43.46 & 43.6767 & -0.2167 \\
\hline 133 & 295.3125 & 308.4113 & -13.0988 & 133 & 42.3077 & 42.1983 & 0.1094 \\
\hline 134 & 286.5625 & 281.7256 & 4.8369 & 134 & 41.5384 & 40.0817 & 1.4567 \\
\hline 135 & 280 & 265.3859 & 14.6141 & 135 & 32.6923 & 32.7148 & -0.0225 \\
\hline 136 & 242.8125 & 271.4428 & -28.6303 & 136 & 27.154 & 27.1563 & -0.0023 \\
\hline 137 & 210 & 305.4505 & -95.4505 & 137 & 50.077 & 50.1289 & -0.0519 \\
\hline 138 & 300 & 302.8641 & -2.8641 & 138 & 55.461 & 55.1454 & 0.3156 \\
\hline 139 & 337.5 & 302.8956 & 34.6044 & 139 & 50.692 & 53.5997 & -2.9077 \\
\hline 140 & 387.5 & 366.2959 & 21.2041 & 140 & 41.123 & 41.0912 & 0.0318 \\
\hline 141 & 406.25 & 427.5018 & -21.2518 & 141 & 37.092 & 37.1099 & -0.0179 \\
\hline 142 & 556.25 & 548.3783 & 7.8717 & 142 & 42.7693 & 44.5296 & -1.7603 \\
\hline 143 & 50 & 67.6593 & -17.6593 & 143 & 45.8031 & 44.8246 & 0.9785 \\
\hline 144 & 90 & 105.7002 & -15.7002 & 144 & 45.8659 & 45.001 & 0.8649 \\
\hline 145 & 195.57 & 204.8164 & -9.2464 & 145 & 46.4085 & 47.6326 & -1.2241 \\
\hline 146 & 318.83 & 267.9977 & 50.8323 & 146 & 44.2302 & 44.2602 & -0.03 \\
\hline 147 & 299.28 & 316.0222 & -16.7422 & 147 & 35 & 35.001 & -0.001 \\
\hline 148 & 265.15 & 323.6264 & -58.4764 & 148 & 43 & 43.0203 & -0.0203 \\
\hline 149 & 260.2 & 280.4688 & -20.2688 & 149 & 47 & 55.0586 & -8.0586 \\
\hline 150 & 235.46 & 217.1329 & 18.3271 & 150 & 38 & 38.0012 & -0.0012 \\
\hline 151 & 121.02 & 158.263 & -37.243 & & & & \\
\hline 152 & 154.032 & 159.2983 & -5.2663 & & & & \\
\hline 153 & 155.044 & 159.9223 & -4.8783 & & & & \\
\hline 154 & 179.203 & 180.1228 & -0.9198 & & & & \\
\hline 155 & 220.21 & 221.2323 & -1.0223 & & & & \\
\hline 156 & 101 & 104.6727 & -3.6727 & & & & \\
\hline 157 & 169 & 197.132 & -28.132 & & & & \\
\hline 158 & 348 & 340.3201 & 7.6799 & & & & \\
\hline 159 & 136 & 236.3055 & -100.306 & & & & \\
\hline 160 & 276 & 289.3115 & -13.3115 & & & & \\
\hline
\end{tabular}

Table 4 Investigation of model validity with other references.

\begin{tabular}{|c|c|c|c|c|c|c|c|c|c|c|c|c|}
\hline \multirow[t]{2}{*}{ Reference } & \multirow{2}{*}{$\begin{array}{l}\text { CS } \\
\text { concentration } \\
(\%)(w / v)\end{array}$} & \multirow{2}{*}{$\begin{array}{l}\text { TPP } \\
\text { concentration } \\
(\%)(w / v)\end{array}$} & \multirow{2}{*}{$\begin{array}{l}\text { CS } \\
M w \\
(k D a)\end{array}$} & \multirow{2}{*}{$\begin{array}{l}\text { DD } \\
(\%)\end{array}$} & \multirow{2}{*}{$\begin{array}{l}\text { CS/TPP } \\
\text { mass } \\
\text { ratio }\end{array}$} & \multirow[t]{2}{*}{$\mathrm{pH}$} & \multirow{2}{*}{$\begin{array}{l}\text { Temperature } \\
\left({ }^{\circ} \mathrm{C}\right)\end{array}$} & \multicolumn{4}{|c|}{ Size(nm) } & \multirow{2}{*}{$\begin{array}{l}\begin{array}{l}\text { Zeta } \\
(\mathrm{mV})\end{array} \\
\text { Predi }\end{array}$} \\
\hline & & & & & & & & Experimental & Predicted & $\begin{array}{l}\text { Error } \\
(\%)\end{array}$ & Experimental & \\
\hline $\begin{array}{l}\text { Grillo et } \\
\text { al. } 57\end{array}$ & 1 & 1 & 27 & 80 & 4 & 4.7 & 25 & 320 & 348.512 & 8.91 & 44.7 & 43.1: \\
\hline $\begin{array}{l}\text { Hussain } \\
\text { and } \\
\text { Sahudin } \\
26\end{array}$ & 2 & 1 & 100 & 85 & 5 & 4.8 & 25 & 227 & 205.8908 & 9.29 & 38 & 35.7 \\
\hline
\end{tabular}

\section{Figures}




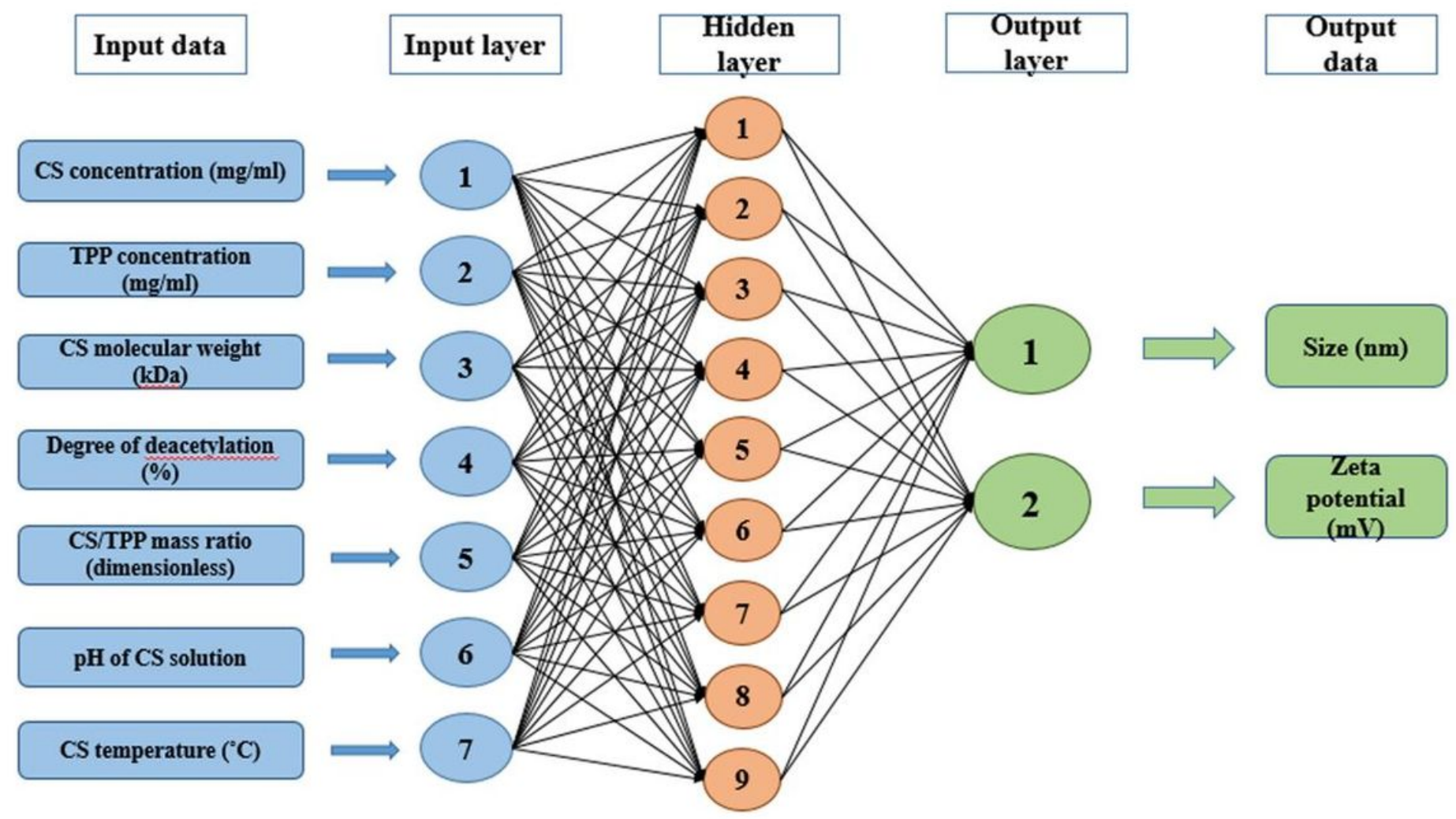

Figure 1

The topology of the ANN structure
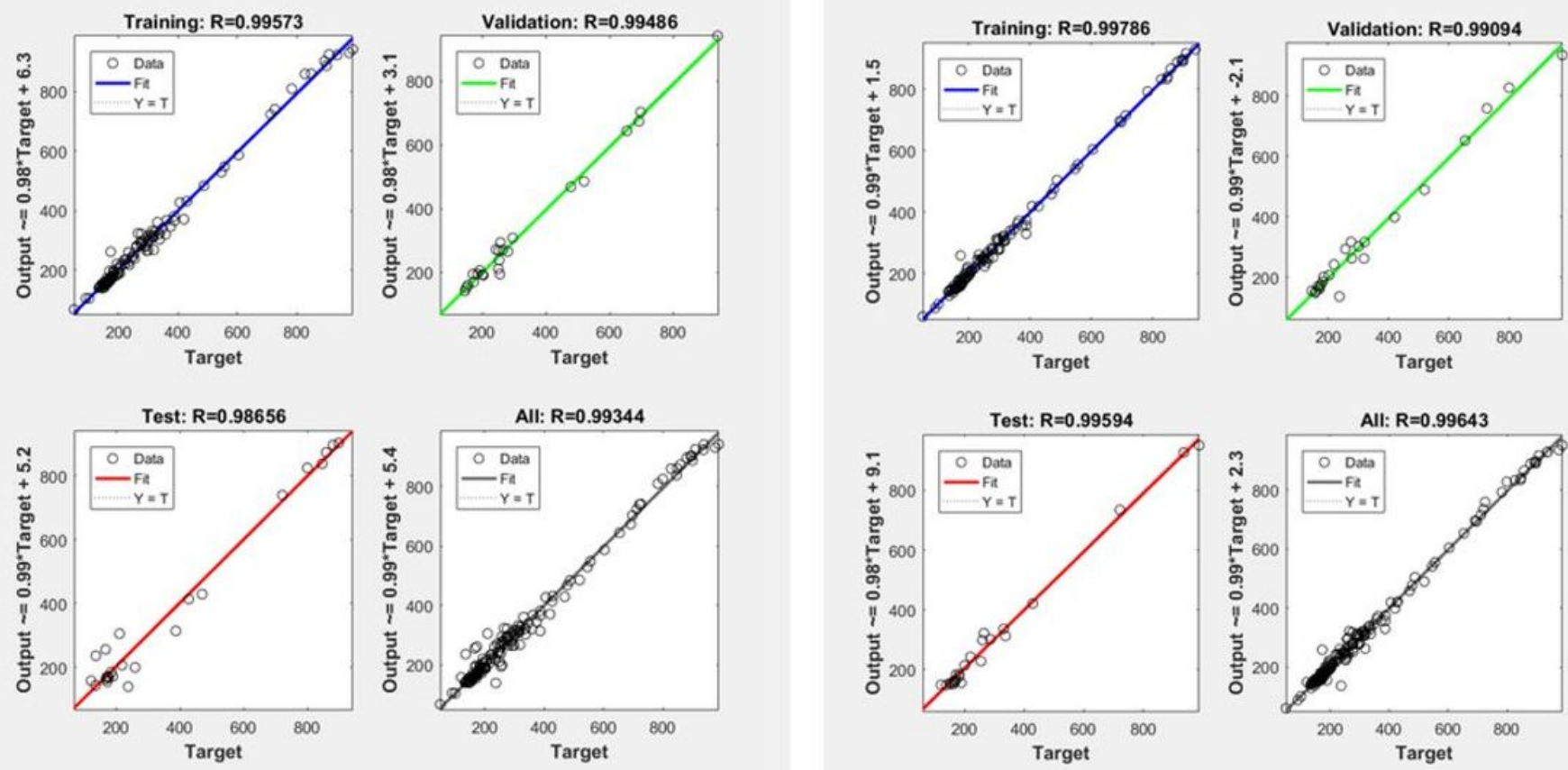

a) Size

b) Zeta potential 
Actual versus predicted particle size from network and regression for training, test, validation, and all data sets.

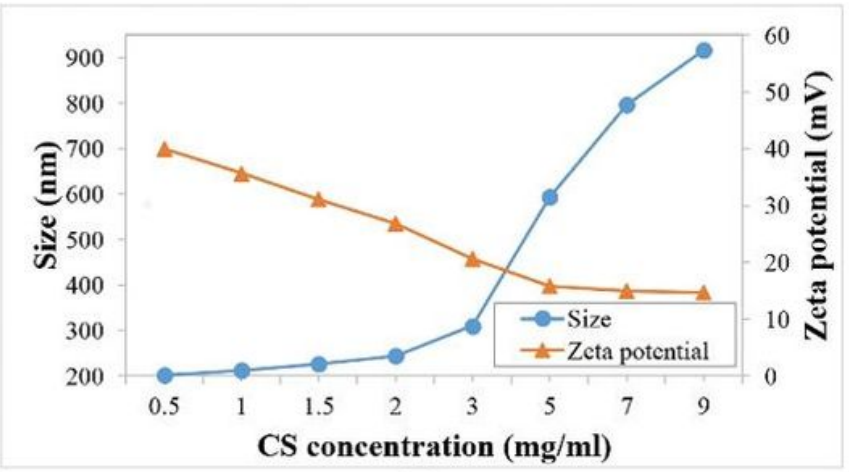

(a)

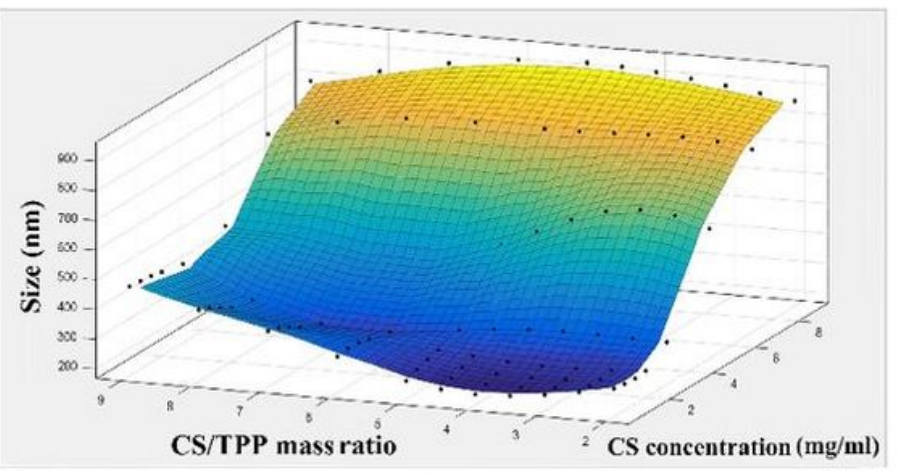

(b)

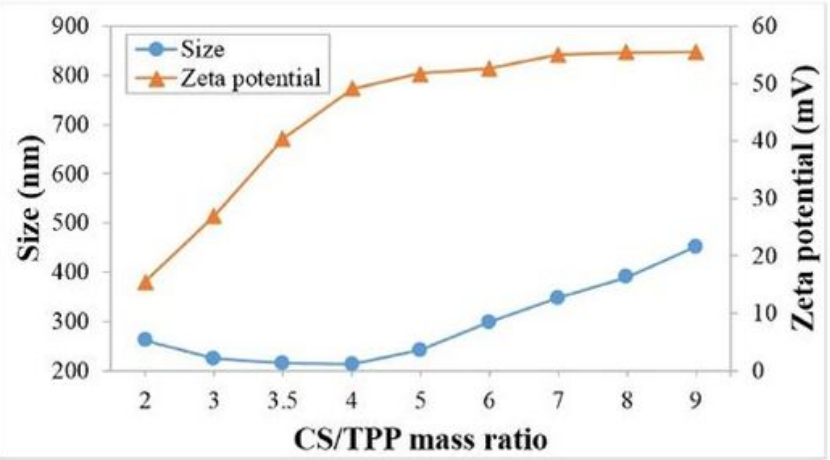

(c)

Figure 3

effect of CS concentration and CS/TPP on the size and zeta potential.

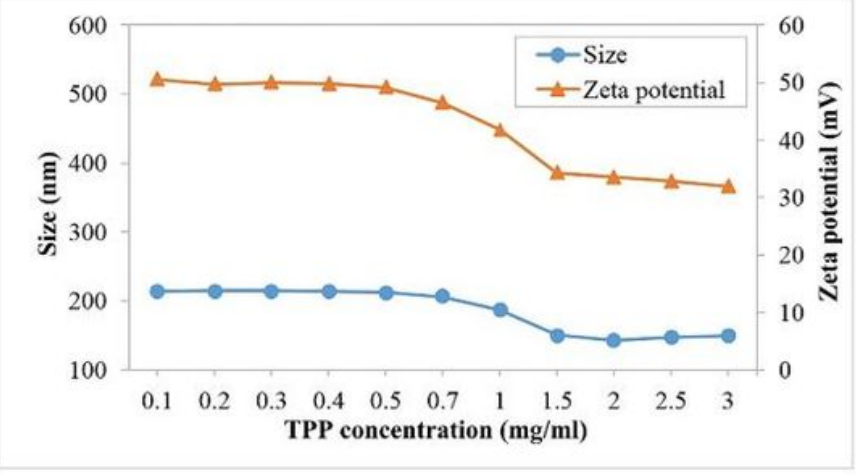

(a)

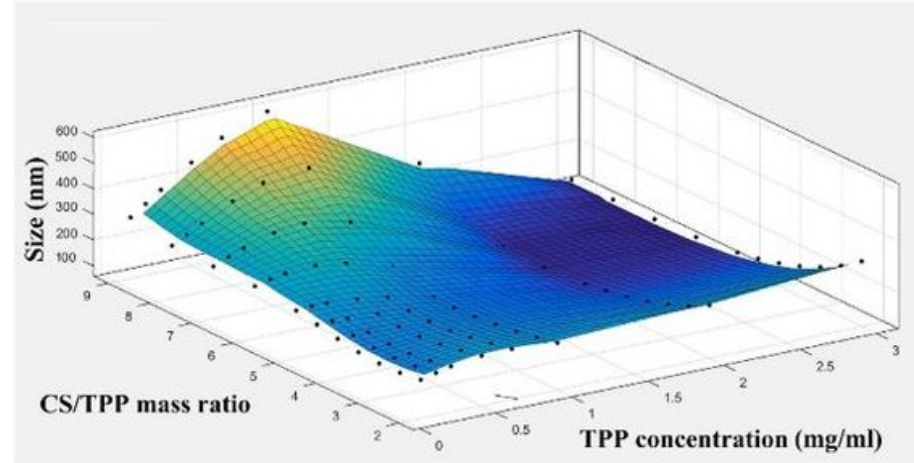

(b)

Figure 4

effect of TPP concentration and CS/TPP mass ratio on the size and zeta potential 


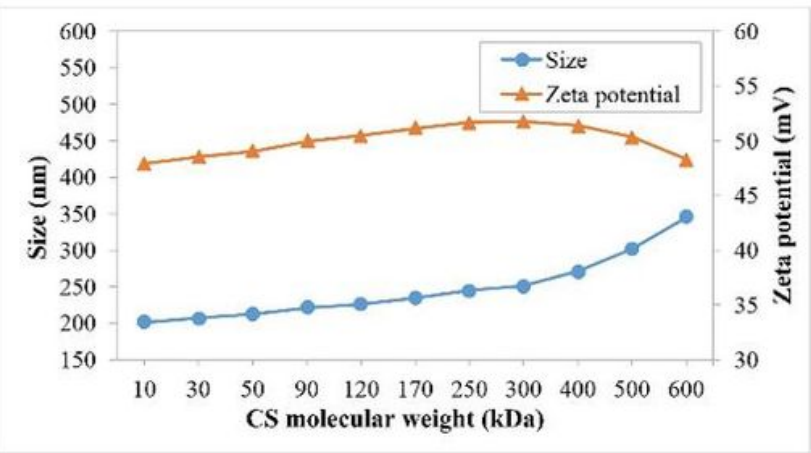

(a)

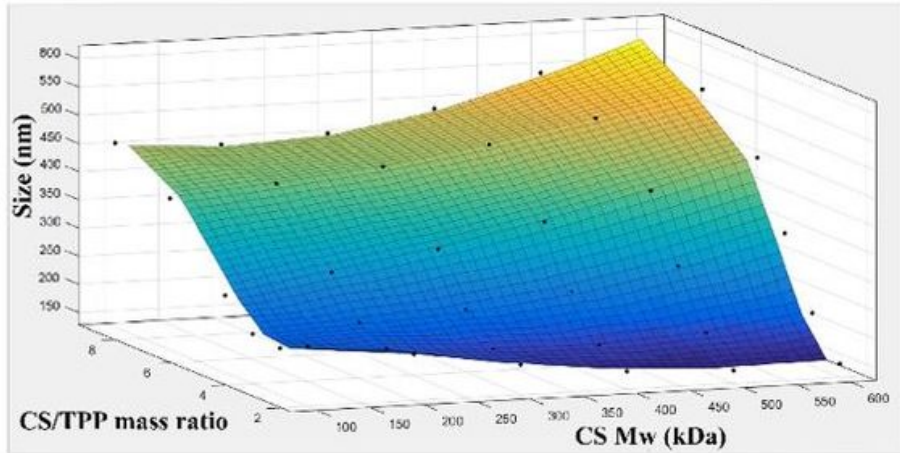

(b)

Figure 5

Effect of CS Mw on the size and zeta potential

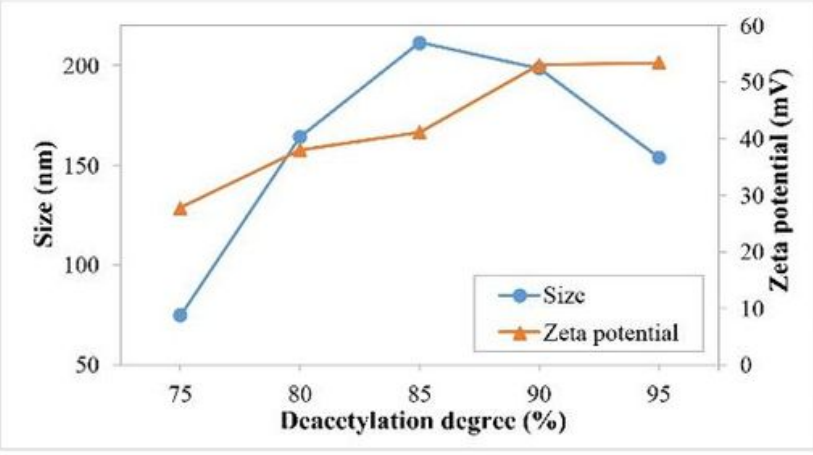

(a)

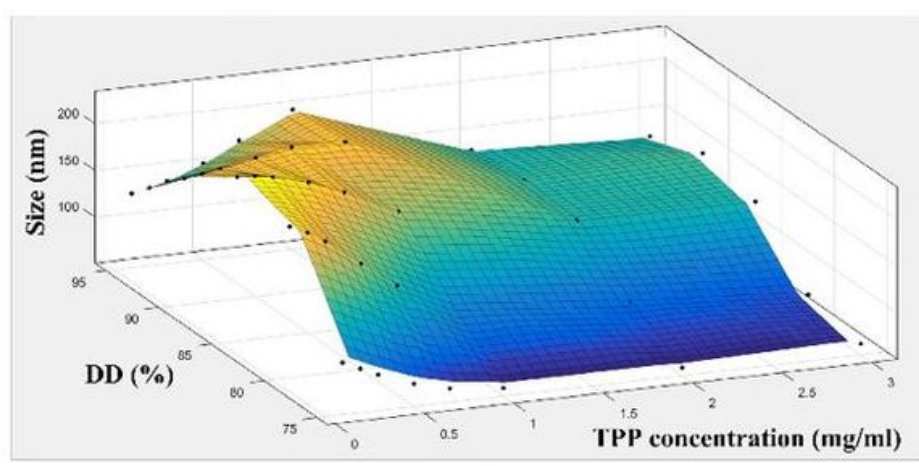

(b)

\section{Figure 6}

Effect of DD and TPP concentration on the size and zeta potential 


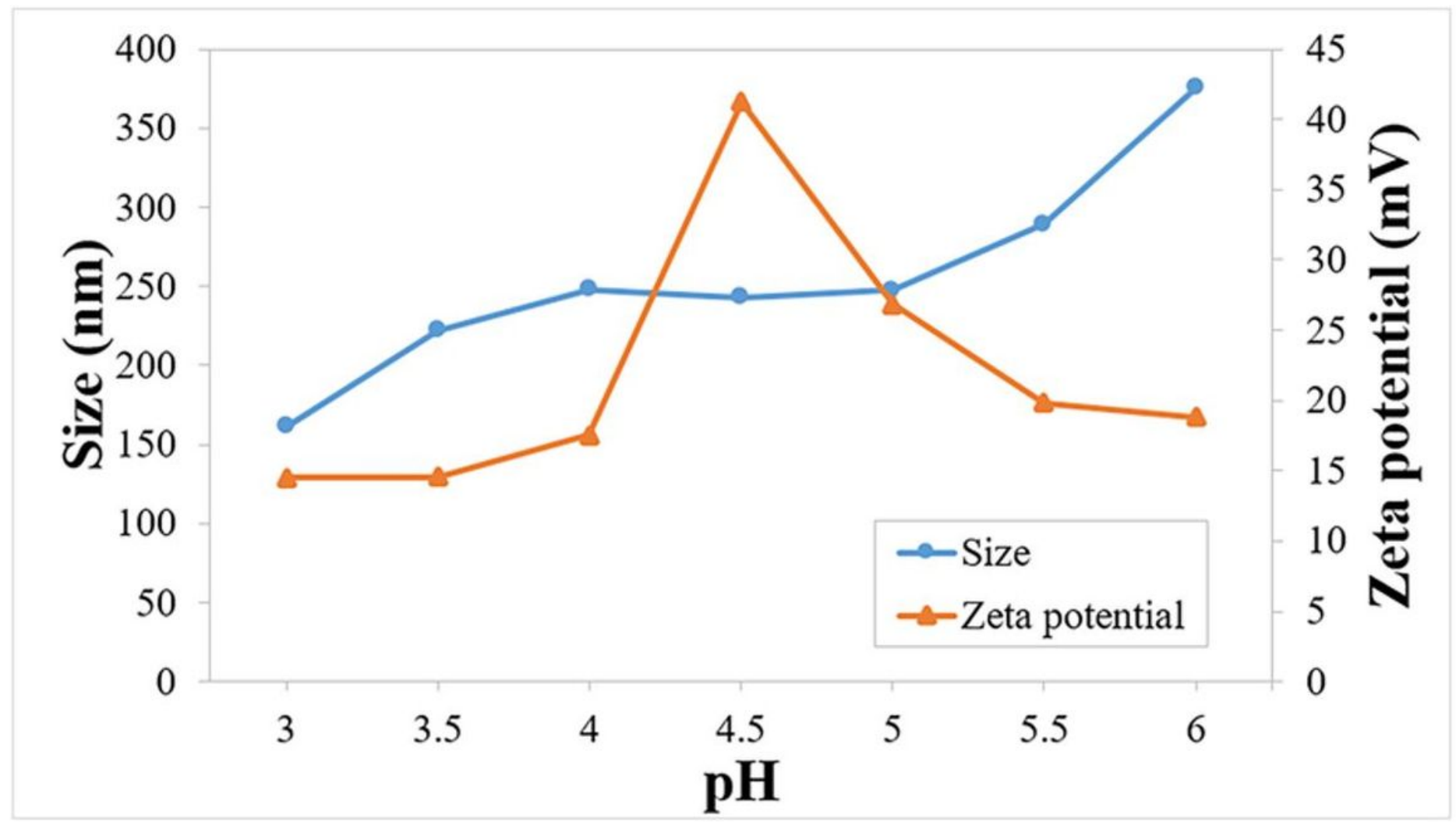

Figure 7

Effect of $\mathrm{pH}$ on the size and zeta potential

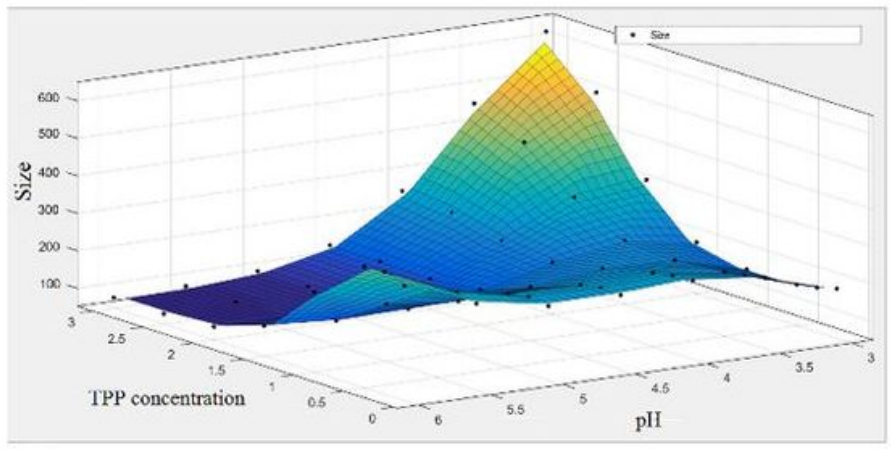

a) TPP concetration and $\mathrm{pH}$

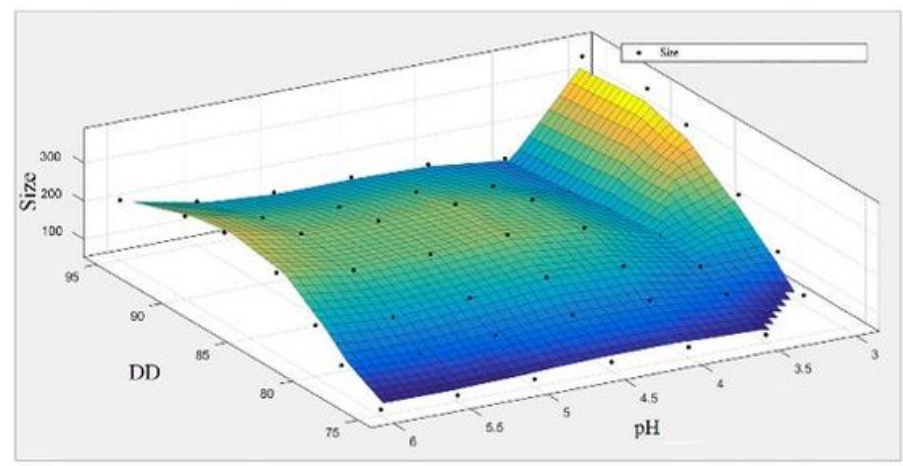

c) Degree of deacetylation and $\mathrm{pH}$

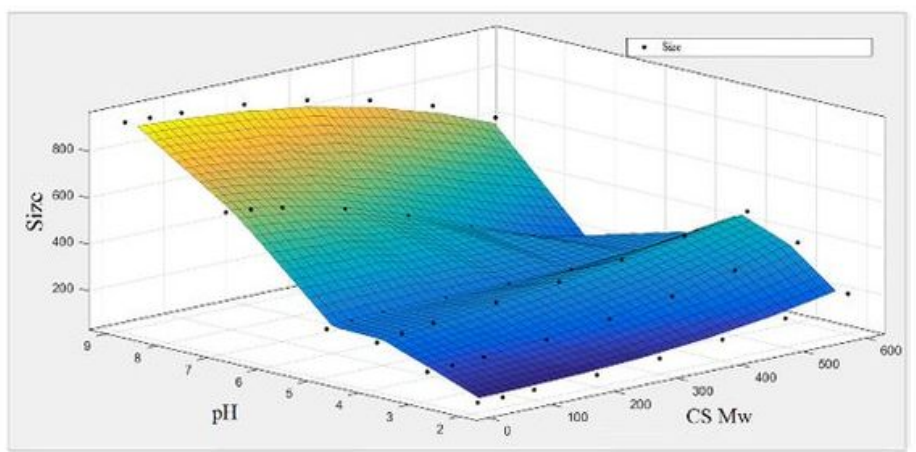

b) $\mathrm{pH}$ and $\mathrm{CS} \mathrm{Mw}$

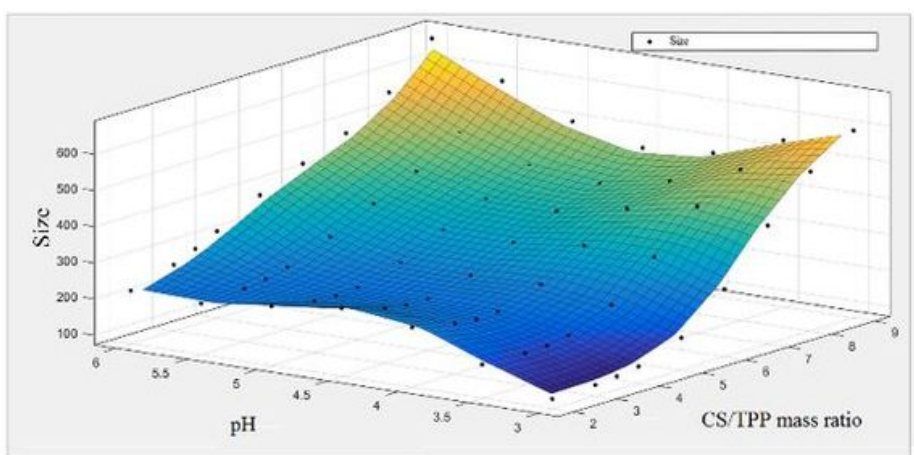

d) $\mathrm{pH}$ and CS/TPP mass ratio 
Effect of interactions between parameters on the size

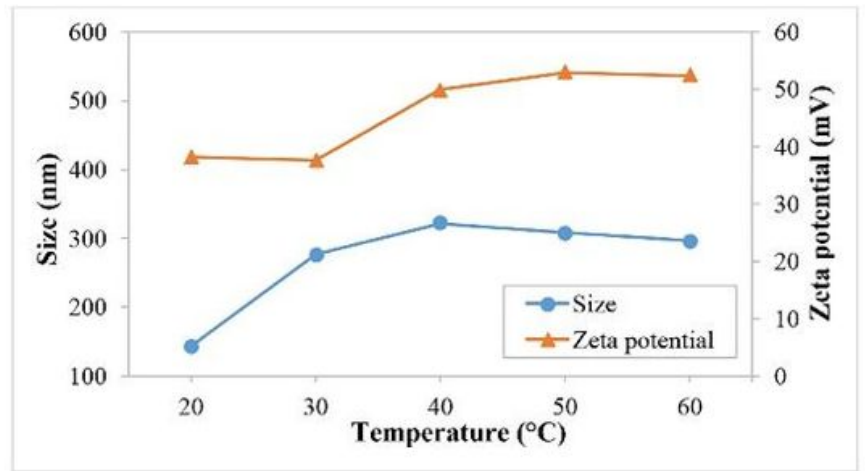

(a)

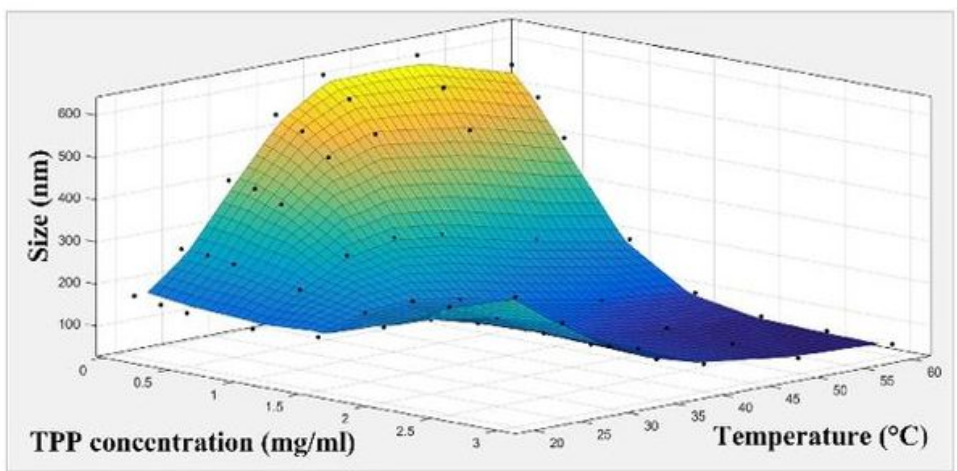

(b)

\section{Figure 9}

Effect of temperature on the size and zeta potential

\section{Supplementary Files}

This is a list of supplementary files associated with this preprint. Click to download.

- GA.jpg 